\title{
Radiation doses along selected flight profiles during two extreme solar cosmic ray events
}

\author{
R. Bütikofer and E. O. Flückiger \\ Physikalisches Institut, Universität Bern, Sidlerstrasse 5, 3012 Bern, Switzerland \\ Received: 27 October 2010 - Revised: 10 January 2011 - Accepted: 16 January 2011 - Published: 13 April 2011
}

\begin{abstract}
The radiation dose rates at flight altitudes may be hazardously increased during solar cosmic ray events. Within the scope of this paper we investigate the total accumulated radiation doses, i.e. the contribution of galactic and solar cosmic rays, during the two extreme solar cosmic ray events on 29 September 1989 and on 20 January 2005 along selected flight profiles. In addition, the paper discusses the consequences of possible solar cosmic ray flux approximations on the results of the radiation dose computations.
\end{abstract}

\section{Introduction}

The radiation dose rates at flight altitudes may be hazardously increased during solar cosmic ray (SCR) events observed at Earth, so-called ground level enhancements (GLEs). The additional contribution caused by SCR is not yet included in most current models for computing the radiation dose rate. The application "Ionization and Radiation Dose Rates in the Earth's Atmosphere" (Bütikofer and Flückiger, 2009), developed under the FP7 project "NMDB" (http://www.nmdb.eu), is capable of taking into account both components: the galactic and the solar cosmic ray flux (GCR and SCR) in near real-time. The calculations of the radiation dose rates are based on the Geant4 (Agostinelli et al., 2003) model PLANETOCOSMICS (Desorgher, 2005). Based on the methods used in this NMDB application we have retrospectively computed the accumulated radiation doses, i.e. the contribution of GCR and SCR, during the two extreme GLEs on 29 September 1989 (GLE 42) and on 20 January 2005 (GLE 69) along selected flight profiles. In addition, the consequences of possible SCR flux approxima-

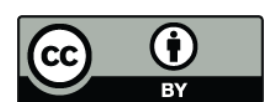

Correspondence to: R. Bütikofer (rolf.buetikofer@space.unibe.ch) tions on the results of the radiation dose computations are investigated.

\section{Characteristics of the ground level enhancements}

The GLEs 42 and 69 are the largest SCR events recorded after the GLE on 23 February 1956. Figure 1 shows the relative pressure corrected count rates of selected neutron monitor (NM) stations during the two GLEs.

The anisotropy, A, of the SCR flux during the two GLEs is illustrated by plotting:

$A=\frac{\Delta N_{S t 1}-\Delta N_{S t 2}}{\Delta N_{S t 1}+\Delta N_{S t 2}}$

where $\Delta N_{S t 1}$ and $\Delta N_{S t 2}$ are the relative GLE count rate increases of the NM stations 1 and 2. The two NM stations are chosen so that their cones of acceptance for SCR point approximately in opposite directions and one station preferably looks into the direction of the apparent source of SCR. The anisotropy plots for the two GLEs using the NM stations Thule and McMurdo are shown in Fig. 2.

\subsection{GLE 42}

The highest NM count rate increase was observed $404 \%$ at the Calgary NM station. The GLE was also observed at low geomagnetic latitude, e.g. Tokyo (effective vertical cutoff rigidity $\sim 11.6 \mathrm{GV}$ ), and by some underground muon detectors, which indicates a hard SCR spectrum and the presence of particles with energies up to $\sim 30 \mathrm{GV}$ (Lovell et al., 1998). The best fit of the SCR spectrum is a modified power law in rigidity (spectral index $\sim-3$ ) where the slope becomes steeper with increasing rigidity (Smart et al., 1991; Lovell et al., 1998). GLE 42 had a complex structure with two maxima at some NM stations. The solar particle flux near Earth had a significant but not extreme anisotropy. At low rigidities $(1-3 \mathrm{GV})$ the anisotropy persisted during the first hour of

Published by Copernicus Publications on behalf of the Arbeitsgemeinschaft Extraterrestrische Forschung e.V. 

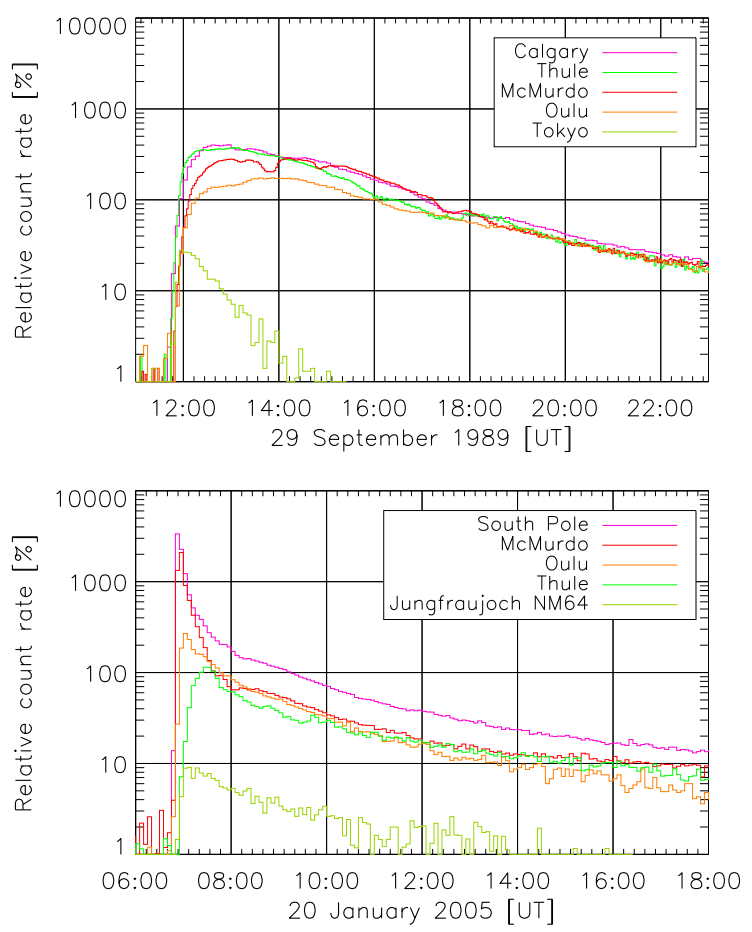

Fig. 1. Relative pressure corrected 2- (Thule and McMurdo during GLE 42) and 5-minute count rates of selected NM stations for 29 September 1989, 1100-2300 UT (GLE 42, top), and for 20 January 2005, 0600-1800 UT (GLE 69, bottom).

the event while at high rigidities the SCR flux became more rapidly isotropic. In addition, GLE 42 was an extremely prolonged event in time; high latitude NM stations still showed an increase in the counting rate of over $10 \% 12$ hours after the GLE onset. The GLE occurred during the maximum activity of solar cycle 22 .

\subsection{GLE 69}

Although near the activity minimum of solar cycle 23, the Sun showed high activity in January 2005. GLE 69 occurred during a Forbush decrease $(\mathrm{Fd})$ that started on 17 January 2005 and showed a maximal drop in the count rate of more than $5 \%$ at high latitude NM stations. The largest count rate increases during GLE 69 in the 1-minute values were observed by the NM stations Terre Adelie (4500\%) and South Pole (more than 5000\%) in the south polar region. During the main phase and the decay phase the spectrum of the SCR flux was relatively soft and could be described by a simple power law in rigidity (spectral index between -7 and -8 ) (Bütikofer et al., 2006). The SCR flux had a large anisotropy during the initial and the main phase of the event. The SCR flux hit the Earth primarily from the southern direction. GLE 69 showed a long duration as well as GLE 42. However, it must also be considered that GLE 69 occurred in the recovery phase of a Fd.
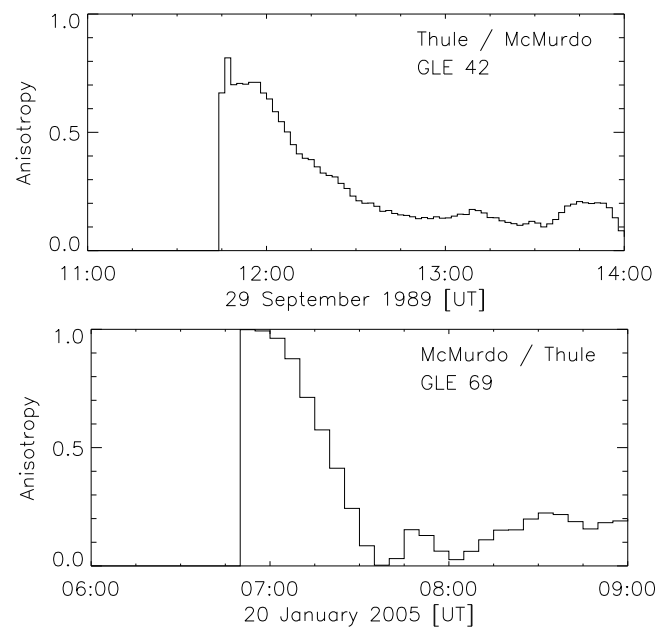

Fig. 2. Anisotropy, $A$, during the GLE 42 (top) and during GLE 69 (bottom) based on the data of the NM stations Thule and McMurdo. For details see the text.

\section{Analysis}

The determination of the accumulated effective radiation dose during a flight is carried out in different steps. First, the time dependent characteristics of the GCR and SCR flux near Earth but outside the geomagnetosphere have to be determined. Then, the transport of the GCR and SCR through the Earth's magnetic field is calculated with the Geant 4 code MAGNETOCOSMICS (Desorgher, 2004). In a next step, the secondary cosmic ray (CR) flux in the Earth's atmosphere caused by primary GCR and SCR is determined for different atmospheric depths at the grid points of a network with the mesh size $5^{\circ} \times 5^{\circ}$ in geographic latitude and longitude by using the Geant 4 code PLANETOCOSMICS (Desorgher, 2005). Then, the effective radiation dose rates at the grid points and at different altitudes are computed by flux to dose conversion factors published by Pelliccioni (2000). Finally the effective dose rates are summed up along selected flight profiles to determine the effective radiation doses.

With this procedure we determined the effective radiation dose rates caused by $\mathrm{CR}$ at typical cruising altitudes as a function of the geographic coordinates, and we determined the effective radiation dose along selected flight profiles. The modulation of the GCR flux in the heliosphere can be described by the so-called force field model (Gleeson and Axford, 1968). This model has only one parameter, the heliocentric potential, $\Phi$. In this work $\Phi$ was applied to the local interstellar GCR spectrum defined by Garcia-Munoz at al. (1975). $\Phi$ was $\sim 1200 \mathrm{MV}$ in September 1989 (Usoskin et al., 2005) and $\sim 595 \mathrm{MV}$ in January 2005 before the Fd (FAA, 2010). The GCR intensity during GLE 69 was set to the Fd level around the GLE onset for the computations of the radiation dose rate caused by GCR. The determination of the SCR characteristics near Earth based on NM data of the 

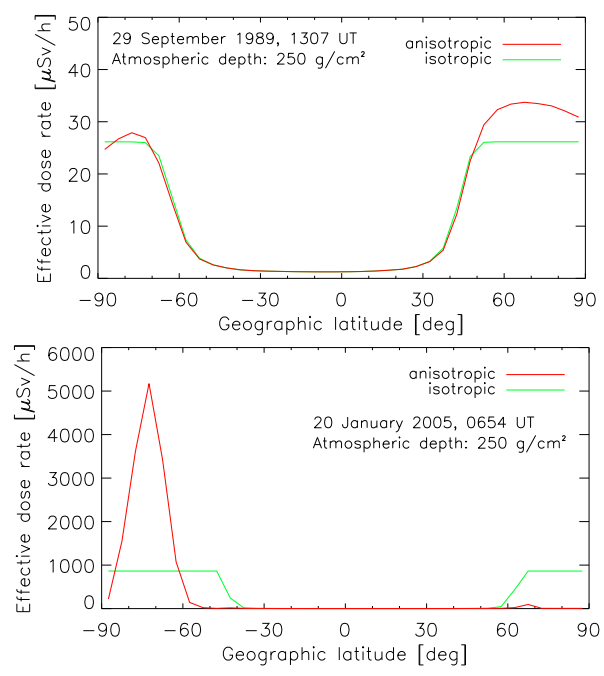

Fig. 3. Effective radiation dose rates at an atmospheric depth of $250 \mathrm{~g} / \mathrm{cm}^{2}(\sim 10.5 \mathrm{~km}$ asl) as a function of geographic latitude with anisotropic SCR flux (red) and isotropic SCR flux (green) during the maximal phase of GLE 42 (top) at longitude $110^{\circ} \mathrm{W}$ and GLE 69 (bottom) at longitude $135^{\circ} \mathrm{E}$.

worldwide network can be carried out e.g. with the method by Smart et al. (1971) and by Debrunner and Lockwood (1980). In this work the characteristics of the anisotropic SCR flux were based on the publications by Smart et al. (1991) and Lovell et al. (1998) for GLE 42 and by Bütikofer et al. (2006) for GLE 69. The SCR spectral form for GLE 69 determined by Bütikofer et al. (2006) agrees with the results by Plainaki et al. (2007); in contrast, the SCR spectrum determined by Bieber et al. (2005) is clearly harder. The characteristics of the SCR flux during the investigated GLEs were used from the above mentioned publications in the rigidity range $1-20 \mathrm{GV}$. For rigidities below $1 \mathrm{GV}$ a constant SCR flux was assumed. The computations were made for anisotropic and isotropic SCR flux. For the isotropic case, an isotropic SCR flux was assumed. The amplitude of the SCR flux, $J_{\text {avg }}$, was determined by integrating over the pitch angle distribution. A constant spectral form, i.e. constant energy dependence during the whole event, for the SCR flux during GLE 42 was used for the isotropic case.

\section{Results}

The computed effective radiation dose rates at an atmospheric depth of $250 \mathrm{~g} / \mathrm{cm}^{2}(\sim 10.5 \mathrm{~km}$ asl) as a function of geographic latitude with anisotropic SCR flux and isotropic SCR flux during the maximal phase of GLE 42 and GLE 69 at the longitude of apparent source direction are plotted in Fig. 3. The difference in the radiation dose rates between the anisotropic and isotropic case is very distinct during GLE 69 where a high north-south anisotropy was present during the
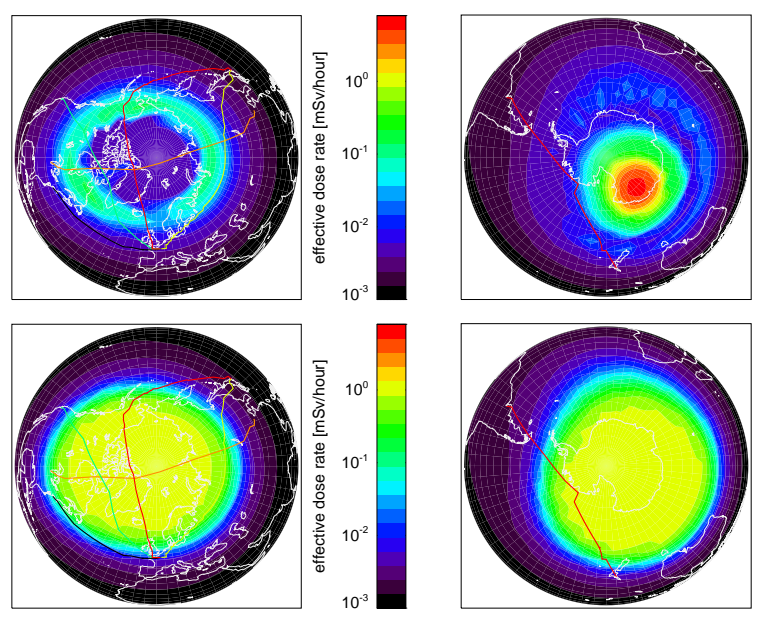

Fig. 4. Computed effective dose rates at an atmospheric depth of $250 \mathrm{~g} / \mathrm{cm}^{2}$ (altitude: $\sim 10.5 \mathrm{~km}$ ) due to combined GCR and SCR during times with maximal SCR flux for GLE 69 with anisotropic (top) and isotropic (bottom) SCR flux at northern (left) and southern (right) hemisphere regions. Flight paths: Chicago-Beijing (orange), Paris-New York (black), Paris-San Francisco (green), ParisTokyo (yellow), Tokyo-Paris (polar route, red), and Buenos AiresAuckland (red).

initial phase of the event. The effective radiation dose rate during the maximal phase of GLE 69 is underestimated by a factor of about 6, if an isotropic SCR flux is assumed at the location with maximal SCR flux entering the atmosphere $\left(72.5^{\circ} \mathrm{S}, 135^{\circ} \mathrm{E}\right)$. The difference between the anisotropic and isotropic case one hour after the GLE onset decreased to $\sim 5 \%$ at the same location.

Figure 4 shows the computed effective radiation dose rates for the anisotropic and isotropic case at typical cruising altitude at northern and southern hemisphere regions due to combined GCR and SCR during times with maximal SCR flux of GLE 69. In addition, the flight paths for the selected flights are plotted. It can be seen from Fig. 4 that none of the investigated flights takes course through the region where the SCR flux entering the atmosphere was maximal (see top right illustration of Fig. 4). Therefore the dose rate along the flight from Buenos Aires, Argentina, to Auckland, New Zealand, during GLE 69 is significantly higher for the isotropic case during the maximal phase.

The accumulated effective radiation doses caused by SCR and GCR were computed for selected actual flight profiles for the anisotropic and isotropic case. The departure time for each flight was adapted so that the dose was maximal (worst case scenario). The results of these computations are listed in Table 1. An approximate relative increase in the radiation dose due to SCR of $0-425 \%$ (anisotropic) and 0-590\% (isotropic) was evaluated relative to the dose level before the onset of GLE 69 in the worst case scenario. The corresponding approximate increase during GLE 42 
Table 1. Computed effective doses caused by GCR only, by SCR only, and combined by GCR+SCR for selected flights during GLE 42 and GLE 69. The departure times of the flights were chosen so that the effective radiation doses for the anisotropic case were maximal (worst case scenario).

\begin{tabular}{|c|c|c|c|c|c|c|c|}
\hline \multicolumn{8}{|c|}{ GLE 42} \\
\hline \multirow[t]{2}{*}{ Flight } & \multirow{2}{*}{$\begin{array}{l}\text { Duration } \\
\text { [min] }\end{array}$} & \multirow{2}{*}{$\begin{array}{l}\text { Max. altitude } \\
\qquad[\mathrm{km}]\end{array}$} & \multirow{2}{*}{$\begin{array}{c}\text { GCR } \\
\text { Sept. } 1989 \\
{[\mu \mathrm{Sv}]}\end{array}$} & \multicolumn{2}{|c|}{$\mathrm{SCR}$} & \multicolumn{2}{|c|}{ GCR+SCR } \\
\hline & & & & $\begin{array}{c}\text { anisotropic } \\
{[\mu \mathrm{Sv}]}\end{array}$ & $\begin{array}{c}\text { isotropic } \\
{[\mu \mathrm{Sv}]}\end{array}$ & $\begin{array}{c}\text { anisotropic } \\
{[\mu \mathrm{Sv}]}\end{array}$ & $\begin{array}{c}\text { isotropic } \\
{[\mu \mathrm{Sv}]}\end{array}$ \\
\hline Buenos Aires - Auckland & 980 & 12.2 & 67 & 204 & 180 & 271 & 247 \\
\hline Chicago - Beijing & 791 & 11.3 & 44 & 112 & 109 & 156 & 153 \\
\hline Paris - New York & 439 & 11.9 & 28 & 43 & 58 & 71 & 86 \\
\hline Paris - San Francisco & 679 & 11.9 & 43 & 144 & 127 & 187 & 170 \\
\hline Paris - Tokyo & 686 & 12.5 & 33 & 64 & 88 & 97 & 121 \\
\hline Tokyo - Paris (polar) & 873 & 11.3 & 50 & 130 & 125 & 180 & 175 \\
\hline Paris - Johannesburg & 598 & 12.1 & 16 & 1 & 1 & 17 & 17 \\
\hline \multicolumn{8}{|c|}{ GLE 69} \\
\hline \multirow[t]{2}{*}{ Flight } & \multirow{2}{*}{$\begin{array}{l}\text { Duration } \\
\text { [min] }\end{array}$} & \multirow{2}{*}{$\begin{array}{c}\text { Max. altitude } \\
{[\mathrm{km}]}\end{array}$} & \multirow{2}{*}{$\begin{array}{c}\text { GCR } \\
\text { Jan. } 2005 \\
{[\mu \mathrm{Sv}]}\end{array}$} & \multicolumn{2}{|c|}{ SCR } & \multicolumn{2}{|c|}{$\mathrm{GCR}+\mathrm{SCR}$} \\
\hline & & & & $\begin{array}{c}\text { anisotropic } \\
{[\mu \mathrm{Sv}]}\end{array}$ & $\begin{array}{c}\text { isotropic } \\
{[\mu \mathrm{Sv}]}\end{array}$ & $\begin{array}{c}\text { anisotropic } \\
{[\mu \mathrm{Sv}]}\end{array}$ & $\begin{array}{c}\text { isotropic } \\
{[\mu \mathrm{Sv}]}\end{array}$ \\
\hline Buenos Aires - Auckland & 980 & 12.2 & 95 & 379 & 555 & 474 & 650 \\
\hline Chicago - Beijing & 791 & 11.3 & 60 & 195 & 317 & 255 & 377 \\
\hline Paris - New York & 439 & 11.9 & 36 & 32 & 53 & 68 & 89 \\
\hline Paris - San Francisco & 679 & 11.9 & 60 & 254 & 355 & 314 & 415 \\
\hline Paris - Tokyo & 686 & 12.5 & 45 & 162 & 254 & 207 & 299 \\
\hline Tokyo - Paris (polar) & 873 & 11.3 & 69 & 262 & 367 & 331 & 436 \\
\hline Paris - Johannesburg & 598 & 12.1 & 20 & 0 & 0 & 20 & 20 \\
\hline
\end{tabular}

was estimated to be 5-335\% (anisotropic) and 5-295\% (isotropic). The largest effective radiation dose for the investigated flights was $\sim 650 \mu \mathrm{Sv}$ (isotropic case) on the flight from Buenos Aires to Auckland during GLE 69. This radiation dose value is almost $40 \%$ higher than the correspondent value for the anisotropic case $(\sim 470 \mu \mathrm{Sv})$, which corresponds to a much more realistic scenario. As mentioned above, the flight path from Buenos Aires to Auckland does not take course close to the direction of anisotropy of the SCR flux. The contribution of SCR along the low latitude flight from Paris to Johannesburg during both GLEs is negligible. During GLE 42 the maximal difference in the total dose (GCR+SCR) between the anisotropic and the isotropic case was $\sim 25 \%$, whereas it was $\sim 40 \%$ during GLE 69 for the investigated flights. The maximal effective dose during one hour at geographic coordinates $72.5^{\circ} \mathrm{S}, 135^{\circ} \mathrm{E}$ (direction of anisotropy) and at $250 \mathrm{~g} / \mathrm{cm}^{2}$ during GLE 69 was $760 \mu \mathrm{Sv}$ (anisotropic case) and $215 \mu \mathrm{Sv}$ (isotropic case).

The comparison of our results (anisotropic case) with computed radiation doses on comparable flights by Lantos and Fuller (2004) and Lantos (2006) shows similar contribution due to GCR but large differences in the contribution of SCR. During GLE 42 the results by Lantos and Fuller (2004) were larger than in this work and the opposite was the case during GLE 69 (Lantos, 2006). Similar outcomes result from the comparison of our computations with the radiation doses determined by Copeland et al. (2008) that computed the radiation doses for flights at high latitudes and at different altitudes during the GLEs 42 and 69. Matthiä et al. (2009) determined the SCR flux during GLE 69 based on NM data of the worldwide network and the resulting accumulated radiation doses along two flights. The SCR flux determined by Matthiä et al. was close to the SCR flux used in this work during the maximum phase of the SCR event, however later during the GLE the two computed SCR spectra differ considerably. In turn, the radiation dose caused by SCR shows large differences. It seems that the reasons for the discrepancy in the computed radiation doses with the different models are namely fundamental differences in the SCR characteristics used.

\section{Conclusions}

The analysis of the two extreme GLEs 42 and 69 has shown that the accumulated radiation dose can significantly increase during large GLEs. The largest accumulated total effective radiation dose over the investigated flights was $\sim 470 \mu \mathrm{Sv}$ for the flight from Buenos Aires to Auckland during GLE 69, i.e. a radiation dose increase of almost $400 \%$ compared to 
the dose caused by GCR alone. A radiation dose of $500 \mu \mathrm{Sv}$ is equal to an accumulated radiation dose of typically $\sim 100$ flight hours with only GCR. The annual hours flown by crew members is $300-900$ hours, i.e. a typical effective radiation dose caused by CR of about $1.5-4.5 \mathrm{mSv}$. If the flight routes during a GLE pass only at low geomagnetic latitudes $\left(<\sim 50^{\circ}\right)$, the total accumulated radiation dose during the flight is marginally increased by the additional SCR flux. The analysis has shown that the dose computation based on an isotropic SCR flux is an insufficient approximation when a large anisotropy in the SCR flux is present, as e.g. during GLE 69. The different models to determine the radiation doses along flights show consistent results for the contribution of the GCR; in contrast, large differences result for the effect by SCR. It seems that notably the determination/description of the SCR characteristics in the different methods is not consistent.

It is not possible to give a practical warning in advance of a GLE for airlines. However, the observation of a GLE occurrence by the worldwide network of NMs could be a resource to initiate measures such as decrease of flight altitude or change the flight path to lower geomagnetic latitudes to minimize the accumulated radiation dose.

Acknowledgements. This research was supported by the Physikalisches Institut, University of Bern, Switzerland, and by the High Altitude Research Stations Jungfraujoch and Gornergrat, Switzerland. We thank the PIs of the NM stations for providing the data used in this work. The information about the flight from Buenos Aires to Auckland were kindly provided by Dr. Vicente Ciancio, La Plata National University, Argentina. The other flight profiles were gratefully received from members of the EURADOS CONRAD WP6 / SG-B.

Edited by: B. Heber

Reviewed by: F. W. Wissmann and another anonymous referee

\section{References}

Agostinelli, S. et al. for the Geant4 Collaboration: Geant4-a simulation toolkit, Nucl. Instrum. Meth. Phys. Res. A, 506, 250-303, 2003

Bieber, J. W., Clem, J., Evenson, P., Pyle, R., Duldig, M., Humble, J., Ruffolo, D., Rujiwarodom, M., and Sáiz, A.: Largest GLE in half a century: Neutron monitor observations of the January 20, 2005 Event, in: Proc. 29th Intern. Cosmic Ray Conf., Pune, India, 3-10 August 2005, 1, 237-240, 2005.

Bütikofer, R. and Flückiger, E. O.: Near real-time determination of ionization and radiation dose rates induced by cosmic rays in the Earth's atmosphere - a NMDB application, in: Proc. 31st Intern. Cosmic Ray Conf., Łódź, Poland, 7-15 July 2009: http: //icrc2009.uni.lodz.pl/proc/html/, last access: 25 January 2011, 2009.

Bütikofer, R., Flückiger, E. O., Desorgher, L., and Moser, M. R.: Analysis of the GLE on January 20, 2005: An update, in: Proc. 20th Europ. Cosmic Ray Symp., Lisbon, Portugal, 5-8 Septem- ber 2006: http://www.lip.pt/events/2006/ecrs/proc/, last access: 25 January 2011, 2006.

Copeland, K., Sauer, H. H., Duke, F. E., and Friedberg, W.: Cosmic radiation exposure of aircraft occupants on simulated highlatitude flights during solar proton events from 1 January 1986 through 1 January 2008, Adv. Space Res., 42, 1008-1029, 2008.

Debrunner, H. and Lockwood, J. A.: The spatial anisotropy, rigidity spectrum, and propagation characteristics of the relativistic solar particles during the event on May 7, 1978, J. Geophys. Res., 85, 6853-6860, 1980.

Desorgher, L.: User guide of the MAGNETOCOSMICS code: http://cosray.unibe.ch/ laurent/magnetocosmics, last access: 25 January 2011, 2004.

Desorgher, L.: PLANETOCOSMICS software user manual: http://cosray.unibe.ch/ laurent/planetocosmics, last access: 25 January 2011, 2005.

FAA, Federal Aviation Administration: http://www.faa.gov, last access: 25 January 2011.

Garcia-Munoz, M., Mason, G. M., and Simpson, J. A.: The anomalous He-4 component in the cosmic-ray spectrum at below approximately $50 \mathrm{MeV}$ per nucleon during 1972-1974, Astrophys. J., 202, 265-275, 1975.

Gleeson, L. J. and Axford, W. I.: Solar modulation of galactic cosmic rays, Astrophys. J., 154, 1011-1026, 1968.

Lantos, P.: Radiation doses potentially received on-board aeroplanes during recent solar particle events, Radiat. Prot. Dosim., 118, 363-374. 2006.

Lantos, P. and Fuller, N.: Semi-empirical model to calculate potential radiation exposure on board airplane during solar particle events, IEEE T. Plasma Sci., 32, 1468-1477, 2004.

Lovell, J. L., Duldig, M. L., and Humble, J. E.: An extended analysis of the September 1989 cosmic ray ground level enhancement, J. Geophys. Res., 103, A10, 23733-23742, 1998.

Matthiä, D., Heber, B., Reitz, G., Meier, M., Sihver, L., Berger, T., and Herbst, K.: Temporal and spatial evolution of the solar energetic particle event on 20 January 2005 and resulting radiation doses in aviation, J. Geophys. Res., 114, A08104, doi:10.1029/2009JA014125, 2009.

Pelliccioni, M.: Overview of fluence-to-effective dose and fluenceto-ambient dose equivalent conversion coefficients for highenergy radiation calculated using the FLUKA code, Radiat. Prot. Dosim., 88, 279-298, 2000.

Plainaki, C., Belov, A., Eroshenko, E., Mavromichalaki, H., and Yanke, V.: Modelling ground level enhancements: Event of 20 January 2005, J. Geophys. Res., 112, A4, 2007.

Smart, D. F., Shea, M. A., and Tanskanen, P. J.: A determination of the spectra, spatial anisotropy, and propagation characteristics of the relativistic solar cosmic-ray flux on November 18, 1968, in: Proc. 12th Intern. Cosmic Ray Conf., Hobart, Australia, 16-25 August 1971, 2, 483-488, 1971.

Smart, D. F., Shea, M. A., Wilson, M. D., and Gentile, L. C.: Solar cosmic rays on 29 September 1989; an analysis using the worldwide network of cosmic ray stations, in: Proc. 22nd Intern. Cosmic Ray Conf., Dublin, Ireland, 11-23 August 1991, 3, 97-100, 1991.

Usoskin, I. G., Alanko-Huotari, K., Kovaltsov, G. A., and Mursula, K.: Heliospheric modulation of cosmic rays: Monthly reconstruction for 1951-2004, J. Geophys. Res., 110, A9, 12108 12117, 2005. 